\title{
ASPECTOS MORFOFONOLÓGICOS NOS PARADIGMAS DOS PRONOMES FORTES E FRACOS
}

\section{MOPHOPHONOLOGICAL ASPECTS IN THE PARADIGMS OF STRONG AND WEAK PRONOUNS}

Mary Aizawa Kato

Universidade Estadual de Campinas

Campinas, São Paulo, Brasil

RESUMO: Considerando que os traços- $\varphi$ podem vir realizados nos pronomes fortes e nos tipos de pronomes fracos (pronomes livres, clíticos ou flexão de concordância), este trabalho propõe que a possibilidade de homofonia (rima) entre eles pode levar à elipse dos pronomes livres fracos, gerando o fenômeno do chamado sujeito nulo pro, e que é na morfofonologia que a criança se pauta para determinar o tipo de língua que ela está aprendendo. Esta proposta embasará a análise do Português Brasileiro com respeito ao Parâmetro do Sujeito Nulo.

PALAVRAS-CHAVE: Pronomes fortes; Pronomes fracos; Concordância; Homofonia; Aquisição.

ABSTRACT: Considering that $\varphi$-features are realized in both pronominal and agreement inflection, this chapter proposes that the possibility of homophony between these elements can lead to the erasure of weak free pronouns creating the so-called little pro, and that it is the morphophonology of such elements that the child uses to determine what type of language he/she is learning. This proposal will also be used to analyze Brazilian Portuguese as far as the Null Subject Parameter is concerned.

KEYWORDS: Strong pronouns; Weak pronouns; Agreement; Homophony; Language; Acquisition. 


\section{INTRODUÇÃO}

A possibilidade de uma língua poder ter ou não sujeito nulo vem merecendo atenção dos linguistas desde o início da década de oitenta com o advento da Teoria de Princípios e Parâmetros (TPP). O tema ainda merece atenção pelos seguintes motivos:

a) o que, efetivamente na morfologia, licencia o sujeito nulo ainda é um mistério, como veremos na seção 1 . abaixo;

b) a pista (ou "trigger") que a criança utiliza para definir sua língua como [+suj nulo] ou [-suj nulo] não pode ser derivada facilmente a partir das teorias sobre licenciamento e identificação do sujeito nulo;

c) o Português do Brasil (PB) pode dar pistas para responder a essas perguntas, justamente por que se pressupõe que esteja mudando de uma língua de sujeito nulo para uma de sujeito não-nulo (DUARTE, 1995), ou para uma língua parcial de sujeito nulo (HOLMBERG e SHEEHAN, 2010).

Usando evidências diacrônicas do Francês Antigo (FA) e do Português Brasileiro (PB) iremos propor que aspectos da morfofonologia dos pronomes pessoais fortes e fracos pode separar as línguas em línguas de sujeito nulo [+SN] e de sujeito não-nulo [-SN] e que é em pistas morfo-fonológicas que a criança brasileira se pauta para definir a gramática que ela está aprendendo.

\section{A RIQUEZA MORFOLÓGICA NO LICENCIAMENTO DO SUJEITO NULO}

A riqueza do paradigma flexional de concordância tem sido uma hipótese consensual nos estudos sobre o parâmetro do sujeito nulo ${ }^{1}$, principalmente no estudo de línguas românicas em oposição a línguas como o inglês, que não admitem sujeitos nulos. Mas Huang (1994) logo trouxe as línguas asiáticas como o chinês e o japonês, sem flexão, que podem igualmente ter o sujeito omitido.

Os dois tipos de língua - com flexão e sem flexão - são tratados, contudo, por Jaeggli e Safir (J\&S) (1989) dentro de uma única hipótese explicativa, de acordo com a qual línguas que apresentam uma morfologia uniformemente flexionada ou uniformemente não-flexionada são as que permitem sujeito nulo. No caso das línguas uniformemente flexionadas, a identificação se dá via concordância e, no caso das línguas não-flexionadas, através de correferência. A análise de $J \& S$ enfrenta problemas com línguas como o alemão, de morfologia flexional uniforme que, todavia, só licencia sujeito expletivo nulo. A explicação

\footnotetext{
${ }^{1}$ V. Taraldsen, 1980; Chomsky, 1981 e muitos outros.
} 
dada pelos autores é estrutural: no alemão Tempo e Concordância estariam dissociados como núcleos.

Rizzi (1986) também propõe essas duas formas de identificação, mas dividindo a definição do parâmetro do sujeito nulo em condição de licenciamento formal e de identificação. De acordo com sua proposta, expletivos nulos só requerem licenciamento formal. $\mathrm{O}$ alemão só permitiria, portanto, licenciamento formal.

Mais recentemente, a diferença entre as línguas tem sido atribuída aos traços morfológicos da flexão e não ao fato de o paradigma ser distintivo para todas as pessoas ou não-marcado para todas as pessoas. Assim, tanto Galves (1993) quanto Rohrbacher (1992) consideram um $A G R$ com o traço [+pessoa] como o responsável pelo licenciamento de pro. O fato de ter havido no PB uma neutralização entre o pronome de segunda pessoa $t u$ e o pronome voce, ${ }^{2}$ forma contraída de Vossa Mercê, resultou na não distinção de segunda e terceira pessoa. Galves atribui o estatuto [-pessoa] a esse paradigma não totalmente distintivo.

(1) mudança de paradigma no PB (adaptado de Duarte, 1993, p. 109)

\begin{tabular}{|c|c|c|c|}
\hline Pessoa & Número & Paradigma 1 & Paradigma \\
\hline 1a. sing & cant-o & cant-o & \\
\hline 2a direta $(\mathrm{tu})$ & sing & canta-s & -------- \\
\hline 2a indireta(você) & sing & canta- $\varnothing$ & canta- $\varnothing$ \\
\hline 3a. sing & canta- $\varnothing$ & canta- $\varnothing$ & \\
\hline 1a. plur & canta-mos & canta-mos & \\
\hline 2a, direta (vós) & plur & canta-is & \\
\hline 2a. indireta (vocês) & plur & canta-m & canta-m \\
\hline 3a. plur & canta-m & canta-m & \\
\hline
\end{tabular}

Em Kato (1996b), considerei I(nflection) como [+pronominal], ou [+pessoa], quando o paradigma apresentasse uma certa homofonia com os pronomes sujeito, fenômeno mais geral de concordância como um fenômeno de rima.
Espanhol
Italiano
Yo habl-o
Io parl-o
El/ella habl-a
Lui/la parl-a

verbos do português terminados em -a Verbos do português terminados em -e ou -i Ela canta

Ele corre

Ela fala

Ele parte

$\begin{array}{llll}\text { Artigos } & \text { Clíticos } & \text { Nomes } & \\ \text { Masc sing } & \mathbf{0} & \mathbf{0} / \mathbf{l o} & \text { menino } \\ \text { Fem. sing } & \mathbf{a} & \mathbf{a} / \mathbf{l a} & \text { menina } \\ \text { Masc sing } & \text { os } & \mathbf{o s} / \mathbf{l o s} & \text { meninos }\end{array}$

${ }^{2}$ Tanto nas regiões em que $t u$ é a forma de tratamento predominante quanto naquelas em que $t u$ e você convivem, a desinência verbal canônica correspondente a $t u$ é apagada (Cf. Scherre et al 2015). 
Femplur as as / las meninas (Grifos meus)

Ao contrário do Romance, o paradigma de concordância das línguas germânicas nada tem de homófono com os pronomes, sendo a morfologia das pessoas do plural homófona à morfologia de número dos nomes. Essa mesma percepção é encontrada em Hoekstra e Hyams (1996), que mostram o caso do holandês, que exibe a concordância -en para todas as pessoas do plural e o morfema $-t$ para o singular, exceto a primeira pessoa. Já o alemão é mais complicado, pois, além da exceção na segunda pessoa do plural, as pessoas do singular apresentam plena distintividade. Mas Rizzi (1982) já havia percebido que o alemão é um tipo de língua que licencia expletivos nulos, embora não admita sujeitos referenciais nulos. As línguas como o alemão, o autor chamou de semipro-drop ${ }^{3}$.

Holandês: "spreken" falar
ik spreek(0)
jij spreekt
hij/zij/het spreekt
wij spreken
julie spreken
zij spreken

Alemão: "spazieren" passear
ich spazier(e)
du spazierst
er/sie spaziert
wir spazieren
ihr spaziert
sie spazieren

Para Hoekstra e Hyams, a segunda pessoa não é crucial para a análise da criança em aquisição pois frequentemente a segunda e a terceira não têm formas distintas (como é o caso do PB). Note-se, porém, que para Duarte (1993) e para Galves (op. cit.) é exatamente a perda da distintividade da segunda pessoa que parece ter afetado o comportamento "pro-drop" do PB.

Neste meu trabalho, considerarei que é efetivamente alguma propriedade da morfologia de concordância que licencia o sujeito nulo e que tanto o comportamento do alemão como do PB serão naturalmente explicados dentro dessa teoria.

\section{PRONOMES FORTES E FRACOS E O FENÔMENO DO REDOBRO}

Com o advento do Minimalismo e a nova visão de Chomsky (1995), na qual é o sujeito que tem os traços interpretáveis e a flexão apenas traços redundantes, não interpretáveis, criou-se um problema para a visão de Rizzi (1986) de que o pro tem de ser identificado pela flexão. Para sair desse impasse, dos tipos de solução apareceram na literatura. Na primeira, na qual me inclui (KATO, 1999), propús que, nas línguas de sujeito nulo, o pronome sujeito é

\footnotetext{
${ }^{3}$ Um exemplo de Cardinaletti (1990) segue em (i), com expletivo, em contraste com (ii) com o pronome referencial. :
}

(I) Gestern wurde (*es) getanzt. (Lit. Yesteray was it danced)

(II) Gestern war *(es) geschlossen. (Lit. Yesterday was it closed) 
afixal, e este aparece como item independente na numeração, com traços- $\varphi$ e de caso $^{4}$. O pronome, quando expresso nessas línguas, é sempre deslocado (DE), externo à sentença, portanto um pronome forte. Kato $(1999 ; 2000)$ adota, em princípio, a tipologia de pronomes de Cardinaletti e Starke (1999), mas em lugar da divisão tripartida em fortes, fracos e clíticos, propõe uma divisão bipartida em fortes e fracos, sendo estes divididos em livres (pronome sujeito do inglês, p. ex.), clíticos (pronome sujeito do trentino, p.ex.) e concordância pronominal (afixo de concordância, no italiano, p. ex.). O pronome forte e o afixo pronominal podem entrar em relação de redobro, como no português europeu (PE) e no espanhol (ESP). Representamos essa posição externa ao IP/TP (Inflectional Phrase/Tense Phrase) como $\Sigma$ P, usando o rótulo usado por Martins (1994) para o sujeito do $\mathrm{PE}^{5}$.
a. [ $\Sigma \mathrm{P}$ EUi [IP com-oi [VP t a pizza ]]]
PE
b. $[\Sigma P Y O i[$ IP com-ii [VP t la pizza $]]]$
ESP

Para atender aos cânones do Minimalismo, a segunda proposta para o pro é formulada como resultado de apagamento do pronome nominativo fraco em PF, funcionando exatamente como um pronome expresso nas línguas [-SN] (HOLMBERG, 2005, ROBERTS, 2010).
$(6)^{\prime}$
a. [ $\Sigma \mathrm{P} \mathrm{EUi}$ [IP eu como [VP t a pizza ]]]
PE

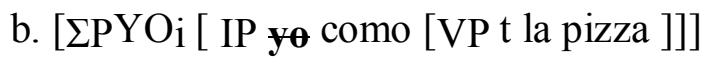
ESP

No $\mathrm{PB}$, com a gramaticalização e forte expansão de você, o pronome reduzido cê parece ter iniciado a introdução de um paradigma de pronomes fracos não totalmente homófonos aos pronome fortes. Nunes (1990) propõe, na verdade, um quasi-paradigma de pronomes fracos $\hat{o}, c \hat{e}$, ei, cês, eis. Assim, de pronomes fracos homófonos aos fortes, o PB passou a pronomes fracos livres, do tipo do inglês, não mais homófonos como no PE, o que impede que se apague o pronome fraco e que o redobro se torne audível.
a. [EUi [ ôi falo espanhol ]]
b. [VOCÊ [ cê fala espanhol ]]
c. [ELE [IP ei fala espanhol ]]

A criação de pronomes fracos livres explica por que em PB o pronome sujeito de $3^{\mathrm{a}}$ pessoa pode ser humano ou não humano, enquanto o pronome expresso de $3^{\mathrm{a}} \mathrm{p}$. no PE é quase categoricamente [+humano] ${ }^{6}$. O pronome, quando expresso no PE, é sempre o pronome forte, sendo a contraparte fraca apagada em $\mathrm{PF}^{7}$.

${ }^{4}$ Cf. Anagnostopoulou \& Alexiadou (1998) para uma visão semelhante.

${ }^{5}$ Veja visão semelhante em Benincà \& Cinque (1985) para o italiano, Soriano (1989) para o espanhol e Barbosa (1997), para o português europeu (PE).

${ }^{6}$ Veja Britto (2000) para um estudo detalhado do problema de redobro no PB.

${ }^{7}$ Mais recentemente venho adotando a hipótese de Roberts (2010) de que pro consiste de elipse de um pronome fraco. 


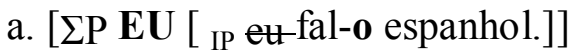
PE
b. [ $\Sigma \mathrm{P}$ TU [ IP tu fala-s espanhol]]
PE


PE

Outro contexto em que temos pronomes fortes é na função predicativa. Se propusermos que os pronomes fortes em posição- $A^{\prime}$ derivam de predicados, fica esclarecido por que o pronome forte em posição A' tem caso default.

(10) a. O culpado fui $\mathbf{e u}_{\text {caso default }}$

b. The culprit was $\mathbf{m e}_{\text {default case }}$

c. Le coupable cést $\mathbf{m o i}_{\text {default case }}$.

(11) a. $\mathbf{E} \mathbf{u}_{\text {default }}$, eu fui o culpado.

b. $\mathbf{M e}_{\text {default }}$, I was the culprit.



Nossa proposta é que esses pronomes fortes em posição-A' se originam de predicados, tais como em construções de aposto:

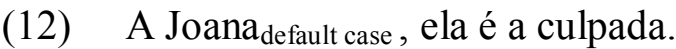

(12)’ a. [тр [DP ela [a Joana]] é a culpada .

b. [торг a Joana ${ }_{i}\left[\right.$ тр $\left[\right.$ ela $\left.\left[t_{i}\right]\right]$ é a culpada $]$

(13) Moi, je suis le meilleur élève de la classe.

(13)’ [TopP Moi $\mathbf{i}_{\mathbf{i}}$ [тр [je [ $\left.\left.\mathrm{t}_{\mathbf{i}}\right]\right]$ suis le meilleur èleve de la classe $\left.]\right]$

A hipótese que queremos sustentar é que para uma língua poder apagar o pronome sujeito fraco é uma condição necessária, embora não suficiente, que o caso default do pronome forte seja o nominativo e que é a homofonia entre os pronomes fortes e fracos que leva ao apagamento do sujeito. Línguas em que o pronome forte é o acusativo ou dativo, como no inglês e no francês, não há possibilidade de se apagar o pronome fraco nominativo por homofonia. Vejam o contraste em [11] acima.

Um fato interessante na criança em fase de aquisição é o aparecimento dos pronomes fortes antes dos fracos ${ }^{8}$. Isso é compreensível pois enquanto os pronomes fortes podem ser adquiridos em uma correspondência direta entre a semântica/pragmática e a fonologia (exs [14]), os fracos são aprendidos a partir da flexão, em especial a flexão de concordância (exs. [15]).
a.Me do. You do. (RADFORD, 1990)
b. Him gone.

(RADFORD, 1990)

\footnotetext{
${ }^{8}$ Kato (2001) mostra que os pronomes fortes aparecem, no início da aquisição, em variação com Nomes e Nomes Próprios.
} 
(15) a. Him, he is gone.

b. Vider la terre moi.

c. Je vais vider la terre, moi.

(LIGHTBOWN,1977: THIMPLI, 1992)

$\mathrm{Na}$ criança brasileira, o pronome fraco livre aparece num quasi jogo de rima $^{9}$ enquanto os fortes aparecem em redobro:

a. $[\hat{\boldsymbol{O}} v \hat{o}] .[\hat{\boldsymbol{E}} l \hat{e}]$
b. $E U,[\hat{o}$ vô]

Vê-se, portanto, que o fenômeno de homofonia (ou rima) pode ser uma relação não só entre pronomes fortes e fracos, mas também entre pronomes fracos e flexão. No japonês, uma língua sem flexão, a homofonia é só entre pronome fortes e fracos:

[KIMI-wa [[TPkimi moo kaeru] no]

VOCÊ-top você já vai voltar?

A relação da homofonia entre pronomes fortes e fracos encontra evidência, ainda, na diacronia de línguas como o francês, que deixou de ser uma língua de sujeito nulo. Roberts (1993, p. 112) mostra estruturas de redobro no Francês antigo, onde havia essa homofonia na primeira pessoa do singular, possivelmente quando já estava deixando de ser uma língua de sujeito nulo.

a. Renars respond: "JOU, je n'irai"

(Coronnement Renart, A.Foulet(ed) 1929,598) Renars responde: "EU, eu não irei.

b. Et JOU je cuit...' (IBID, 1616)

e EU eu acredito

Dufresne e Dupuis (1996), entretanto, mostram que no francês de Moignet já havia competição entre o nominativo "default" com o dativo:

(18) a. Et lors en irons MOI et VOS aprés le chevalier e depois iremos ME e VOS atrás do cavaleiro

b. dist li rois à Boort qu'il venist a cort, LUI et sa compaignie disse o rei a Boort que ele viesse à corte, LHE e sua companhi

No francês de hoje, quando há redobro, é sempre o dativo que aparece no pronome forte:

a. MOI, je cuit ... $\mathrm{EU}_{\text {dat }}$ eu acredito

b. LUI, il vient toujours. ELE $_{\text {dat }}$ ele vem sempre

\footnotetext{
${ }^{9}$ Fazemos em transcriçãoo fonética a fala da criança para ficar mais clara a percepção infantil.
} 
No PB, o fenômeno se manifesta como no francês antigo antes do pronome forte passar a dativo. Enquanto era uma língua de sujeito nulo, não se ouvia o redobro, pois o sujeito nominativo era apagado, nulo. Quando deixou de ser uma língua de sujeito nulo, começamos a ouvir o redobro como no francês antigo, nos exemplos em (17):

a. Você, 'cê não me pega!

b. Eu , [ô] sinto demais isso, né?

A formação de um paradigma de pronomes fracos no $\mathrm{PB}$, não exatamente homófonos aos pronomes fortes, favorece a manutenção dessas formas fracas na saída em PF.

Como já dissemos, a homofonia não se limita ao domínio dos pronomes fortes e fracos, mas atinge também o domínio da flexão de concordância, aqui considerada como constituída de pronomes fracos. A idéia intuitiva de que temos nas línguas de sujeito nulo uma concordância pronominal está no fato de que as línguas de sujeito nulo têm uma morfologia flexional que pode ser homófona à dos pronomes fracos, o que fica claro ao observar crianças em aquisição, como vimos no exemplo [15] acima e no exemplo [21] abaixo, do espanhol, supondo que o pronome fraco é apagado:

a. YO, (yo) hablo

b. ELLA, (ella) habla

Note-se que o alemão, embora provido de um rico sistema de flexão, não licencia sujeitos nulos - exceto o expletivo - justamente porque não exibe o tipo de homofonia do espanhol.

Resumindo as línguas até aqui vistas, temos a seguinte tabela:

\begin{tabular}{|c|c|c|c|c|c|c|}
\hline & $\mathrm{DE}$ & & SUJ & & AGR & \\
\hline Japonês & fortes & $=$ & fracos & $\varnothing$ & AGR & (total homofonia) \\
\hline Espanhol/Italiano & fortes & $=$ & fracos & $=$ & AGR & (total homofonia) \\
\hline Finlandês & fortes & $=$ & fracos & $\neq$ & AGR & (parcial homofonia) \\
\hline $\mathrm{BP}$ & fortes & $\neq$ & fracos & $=$ & AGR & (parcial homofonia) \\
\hline Alemão & fortes & $=$ & fracos & $\neq$ & AGR & (parcial homofonia) \\
\hline Inglês/Francês & fortes & $\neq$ & fracos & $\neq$ & AGR & (0 homofonia) \\
\hline
\end{tabular}

O que a Tabela acima mostra é que a homofonia entre pronomes fortes e portadores de traços- $\varphi$ é crucial para a possibilidade de sujeitos nulos. Quando há total homofonia a língua licencia tanto sujeitos nulos referenciais quanto nãoreferenciais. Se o pronome fraco exibir homofonia ou com os pronomes fortes ou com a flexão, a língua será do tipo de sujeito nulo parcial. Se nenhuma homofonia estiver presente, nenhum tipo de sujeito nulo será permitido na língua.

Minha análise é reforçada pela hipótese de Hoekstra a Hyams (1996) de que o que licencia a natureza pro-drop de uma língua é a natureza da flexão: a) se INFL é rico mas manifesta apenas número, como o holandês, ele não licencia o 
apagamento do sujeito; b) se INFL manifesta distinção de pessoa, ele licencia o apagamento. Mas, ao contrário da análise de Hoekstra e Hyams, que é baseada apenas em critério morfológico, a nossa é também pautada em critério fonológico. Para Hoekstra e Hyams a segunda pessoa não é crucial para a criança fixar o parâmetro, pois a segunda é frequentemente indistinguível da terceira pessoa, caso do PB. Mas lembremos que no Romance, todavia, a segunda pessoa do singular é homófona na flexão do perfectivo. Por outro lado, a terceira pessoa não exibe homofonia, mas como se pode observar em [23], existe a homofonia com o sujeito DP de terceira pessoa:

\section{(23) La ragazza parla.}

Para concluir nossa análise, temos que ver como relacionar as restrições nas interfaces. Até aqui vimos que a possibilidade de apagamento do sujeito pronominal fraco pode ser predita por propriedades morfofonológicas:

(a) o apagamento dos pronomes fracos depende da sua homofonia com seus controladores (DE ou AGR);

(b) o apagamento dos pronomes sujeitos referenciais requer homofonia com os dois tipos de controladores quando há AGR, caso do italiano, e com o único controlador, o pronome forte, quando não há AGR, caso do japonês;

(c) se existe AGR, e a homofonia funciona com um dos controladores, mas não com ambos, os pronomes referenciais não podem ser nulos.

Nossa análise nos leva a reforçar a nossa hipótese inicial de que estruturas de redobro são o input crucial para a seleção da gramática pela criança. Além disso, podemos afirmar que pistas semânticas podem ser usadas por ela naquilo que os autores vêm chamando de "semantic bootstrapping hypothesis" antes de fixar o parâmetro via a morfossintaxe.

\section{OS SUBTIPOS DE LÍNGUAS DE SUJEITO NULO}

Vimos acima que o japonês e o Português Europeu (PE) constituem línguas com homofonia total, e que licenciam sujeitos nulos referenciais e nãoreferenciais, apesar de serem aparentemente tão diferentes quanto à identificação do sujeito nulo referencial, o primeiro identificado via antecedente e o segundo via concordância. O primeiro tipo tem sido chamado de língua de sujeito nulo radical e o segundo de língua de sujeito nulo consistente. Na verdade, o japonês tem o mesmo mecanismo de identificação do sujeito nulo quando, no PE, temos a terceira pessoa.
a. O Pedro ${ }_{i}$ está dizendo [que $\boldsymbol{0}_{i}$ gosta de pizza].
PE
b. Pita ${ }^{-}$-wa $\left[\mathbf{0}_{\mathbf{i}}\right.$ pizza ga suki-da] to yute-iru. 
Já as línguas sem homofonia, isto é [-SN] . como o inglês não admitem controle em ambiente finito, exigindo a repetição do sujeito referencial:

Peter is saying [ that he likes pizza].

O PB vernacular, contudo, se comporta como o inglês manifestando o sujeito referencial ${ }^{10}$, o que demonstra a mudança em progresso para língua [-SN] parcial, já que mantém o sujeito nulo quando não-referencial, em qualquer circunstância:


PB
b. O Pedro disse [que 0 está chovendo].
PB PE

Nesse sentido o PB se assemelha a línguas como o islandês, os chamados semi-pro-drop, que se caracterizam por ter sujeitos pronominais quando referenciais e nulos quando não referenciais. O PB vernacular se caracteriza por não respeitar o Princípio "Evite Pronome". de Chomsky (1981), pois a manifestação do pronome sujeito se dá em qualquer pessoa e não apenas em ambiente controlado:

(27) a. Eu acho que eu passaria por causa da base que eu tinha.

b. Vocês dizem isso porque vocês são jovens.

c. Ela ficou solteira porque ela quis.

(apud KATO e DUARTE, 2017)

Quanto aos não-referenciais, o PB tem não só expletivos nulos, mas também sujeitos indefinidos e genéricos nulos, tal como o islandês, língua semiprodrop (SIGUR $\delta S S O H N$; EGERLAND, 2009).

a. Sinne ei muut vapehtoisesti

Expl. não.3SG move voluntariamente

'Não se move pra lá voluntariamente.'

b. Í pessari fjölskyldu drekkuv pú bara ekki áfengi

nesta família pode vc beber alcool nao

' Nesta família você simplesmente não bebe alcool.'

c. Deir segja að pað rigni a'morgun.

Eles dizem que ele chove na manhã

'Eles dizem que vai chover esta manhã.'

d. Menn na'ðu bo' fanum um kvo“ldið.

homens pegaram o culpado de manhã

'Pegaram o culpado esta manhã.'

Holmberg and Sheehan (2010) classificaram o PB como uma língua de sujeito nulo (SN) parcial como o finlandês, mas Kato e Duarte (2017) acreditam não ser esse o caso, por haver mais dissimilaridades do que semelhanças.

\footnotetext{
${ }^{10}$ Mas nós, os letrados, que adquirimos o sujeito nulo via instrução escolar, usamos o nulo quando escrevemos.
} 
Lembremos que a opcionalidade no PB tem a ver com a escolarização. Na gramática nuclear da criança só há pronomes expressos.

Nota-se, nessa breve comparação com o PB e outras línguas parcialmente homófonas que há uma certa correspondência entre homofonia de elementos portadores de traços- $\varphi$ e tipo de línguas em relação ao Parâmetro do Sujeito Nulo.

\section{CONCLUSÃO}

A definição do que vem sendo chamado de AGR [+pronominal] como um afixo com vogal harmônica ao pronome fraco lembra da velha idéia de Givón (1978) de que a concordância é a gramaticalização do sujeito pronominal, quando o Tópico (ou o que vimos chamando DE se gramaticaliza como Sujeito. Tal gramaticalização pressupõe certo grau de homofonia entre o pronome sujeito e o afixo de Concordância. Quando a Concordância é morfofonologicamente independente do paradigma pronominal, a elipse pronominal não pode ocorrer. Este é o caso do alemão, que apesar da riqueza flexional não admite pronomes nulos, exceto o expletivo.

\section{REFERÊNCIAS}

AGNOSTOPOUlU, E.; A. ALEXIADOU,A. Parametrizing AGR: word order, V-movement and EPP-checking. Natural Language and Linguistic Theory. Springer n. 16, 1998, p. 491-540.

BARBOSA, P. Subject position in the null subject languages. Seminários de Linguística, n. 1, p. 39-63. Faro: Universidde de Algarve. UCEH. 1997.

BENINCÁ, P. E G.CINQUE. Lexical subjects in Italian and the pro-drop parameter. Trabalho apresentado em Comparative Generative Grammar Fiesta. Salzburg. 1985

BRITTO, H. Syntactic codification of categorical and thetic judgmnents in Brazilian Portuguese. In: KATO, M.A.; NEGRÃO, E.V. (Orgs). Brazilian Portuguese and the Null Subject Parameter. Frankfurt: Vervuert-IberoAmericana, 2000, p. 195-222.

CARDINALETTI, A. Impersonal Constructions and Sentential Arguments in German. Padua: Unipress. 2000.

; STARKE, M. The typology of structural deficiency: a case study of the three classes of pronouns. In: RIEMSDIJK, H. v. (ed.), Clitics in the Languages of Europe. Berlin -New York: Mouton de Gruyter, 1999, p. 145-233.

CHOMSKY. N. Lectures on Government and Binding. Dordrecht: Foris, 1981. 
CHOMSKY. N. The Minimalist Program. Cambridge, Mass: The MIT Press. 1995.

DUARTE, M.E. Do pronome nulo ao pronome pleno: trajetória do sujeito no português do Brasil. In: ROBERTS, I ; KATO, M.A. (Orgs). Português Brasiliero: uma viagem diacrônica. São Paulo, Contexto . 2018, p. 107-128.

DUARTE, M.E. A Perda do Princípio "Evite pronome" no Português Brasileiro. Campinas, 1995. UNICAMP: Tese de Doutorado.

DUFRESNE.M; DUPUIS, F. Modularity and the reanalysis of the French subject pronoun.

GALVES, C. O enfraquecimento da concordância no Portugues Brasileiro.

In: ROBERTS ; KATO (eds). Português Brasiliero: uma viagem diacrônica. São Paulo: Contexto . 2018, p. 387-408.

JAEGGLI ,O.; SAFIR, K. The null subject parameter and parametric theory. In: JAEGGLI, O; SAFIR, K (Orgs). The Null Subject Parameter. Dordrecht: Kluwer. 1989.

HOEKSTRA.T.; HYAMAS, N. The syntax and interpretation of dropped categories. In: CAMACHO, J; CHOUERI, L; WATANABE, M (Orgs) The Proceedings of the Fourteenth West Coast Conference on Formal Linguistics. Stanford: Center for the Study of Language and Information. 1996.

HOLMBERG, A. Is there a little pro? Evidence from Finnish. Linguistic Inquiry. Cambridge, Mass., n. 36, 2005, p. 533-564.

HOLMBERG, A.; SHEEHAN, M. Control into finite clauses in partial nullsubject languages. In: BIBERAUER, P; Holmberg, A; ROBERTS, I; SHEEHAN, M (Orgs.) Parametric Variation. [s.1.], 2010, p. 125-152.

HUANG. T.J. On the distribution and reference of empty pronouns. Linguistic Inquiry, n. 15, [s.1.], 1984, p. 531-574.

HYAMS, N. The underspecification of functional categories in early grammars. In: CLAHSEN, H; HAWKINS, R (eds). Generative Approaches to first and Second Language Acquisition. Amsterdam: John Benjamins. 1994, p. 91-127.

KATO, M.A. Strong pronouns and weak pronominals in the null subject parameter. Probus, n. 11, 1, [s.1.], 1999, p. 1-37.

KATO, M.A. The partial pro-drop nature and the restricted VS order un Brazilian Portuguese. In: KATO, M.A; NEGRÃO, E.V (Orgs)., 2000, p. 223-258.

KATO, M.A. Nomes e pronomes na aquisição. Letras de Hoje. Porto Alegre: PUCRS, n. 36,3, 2001, p. 101-112. 
KATO, M.A; DUARTE, M.E. O sujeito no Português Brasileiro e sua tipologia. In: PILATI, E; SALLES, H, L; NAVES, R (Orgs). Novos Olhares para a Gramática do Portuguê Brasileiro. Campinas, Ed. Pontes. 2017, p. 13-42.

KATO, M.A; NEGRÃO, E.V. Brazilian Portuguese and the Null Subject Parameter. Frankfurt: Vervuert-IberoAmericana. 2000.

MARTINS, A M. Clíticos na História do Português. Lisboa, Estremeda: 1994. Universidade de Lisboa: Tese de doutorado.

NUNES, J. Nominative pronoun reduction, in Brazilian Portuguese. Annapolis, Maryland: 1990. University of Maryland. Ms..

RADFORD, A. Syntactic Theory and the Acquisition of English Syntax. Oxford: Basil Blackwell. 1990.

RIZZI, L. Issues in Italian Syntax. Dordrecht: Foris. 1982.

RIZZI, L. Null objects and the theory of pro. Linguistic Inquiry. Cambridge, Mass. n. 17,1986 , p. 501-558.

ROBERTS, I. Verbs and Diachronic Syntax. Dordrecht: Kluwer. 1993.

ROBERTS, I. A deletion analysis of null subjects. In: BIBERAUER, T; HOLMBERG, A; ROBERTS, I; SHEEHAN, M (Orgs.) Parametric variation: Null subjects in Minimalist Theory. Cambridge: Cambridge University Press. 2010.

ROBERTS, I;KATO, M.A. Português Brasileiro: uma viagem diacrônica. São Paulo, Contexto, 2018.

ROHRBACHER. B. English AUX ${ }^{\wedge}$ NEG , Mainland Sacandinavian $\mathrm{NEG}^{\wedge} \mathrm{AUX}$ and the theory of $\mathrm{V}$ to I raising. Proceedings of the 22nd Western Conference on Linguistics. (WECOL, 92). 1992.

SCHERRE, M. et al. Variação dos pronomes TU e VOCÊ. In: MARTINS, M.A; ABRAÇADO, J (Orgs.) Mapeamento sociolinguístico do português brasileiro. São Paulo: Contexto, 2015, p. 133-155.

SIGURSSSON, H. Á; EGERLAND, V. Impersonal null-subjects in Icelandic and elsewhere. Studia Linguistica. Wiley, 2009, p. 158-185.

SORIANO, O. Strong pronouns in null subject languages and the avoid pronoun principle. MIT Working Papers in Linguistics. Cambridge, Mass. Vol 11, 1989, p. 228-239. 
TARALDSEN, K. On the nominative island condition, vacuous application, and the that-trace filter. Indiana: Linguistics Club. 1980.

Mary Aizawa Kato

Mary.Kato@gmail.com

Recebido em: 22 jan. 2018

Aceito em: 10 maio 2018

Publicado em: 1 out. 2018 